\title{
Flow-induced crystallization of polyethylene melts
}

\author{
M. R. MACKLEY, F. C. FRANK, A. KELLER \\ H. H. Wills Physics Laboratory, University of Bristol, Bristol, UK
}

In situ optical observations have been made of the way polyethylene melt can crystallize whilst subject to certain longitudinal velocity gradients. In general crystallization is seen to occur as the generation of fibres 5 to $50 \mu \mathrm{m}$ in diameter. Hydrodynamic considerations lead to the conclusion that the externally applied velocity field is responsible for the nucleation of the fibrous crystals, subsequent growth is then influenced by both the local streaming of polymer melt around the growing tip of the fibre and the external velocity field. The effect enhanced pressure has on flow induced crystallization is also examined.

\section{Introduction}

The current work forms the continuation of earlier studies on the effect of elongational flow on chain orientation and on orientation induced crystallization in polyethylene, both from solution and melt. Previously, the elongational flow field was achieved by an arrangement of mutually opposed jets and the effects occurring in the region of the jet orifices were observed in situ through a microscope. The work on solutions is subject to a comprehensive publication [1] and of two subsequent shorter papers $[2,3]$. The melt case features in a preliminary paper [4] describing the experimental arrangements together with some observations made while the melt was forced through two opposing jets. The purpose of the present paper is to report further observations on the melt which highlight some common underlying physical principles which are of relevance in terms of both scientific and technical interest. The experiments fall into two groups: (1) formation of fibrous crystals in the flowing melt, and (2) crystallization effects observed between double jets where blockage could occur as a result of enhanced pressure. For reasons which will be apparent, the observations of Section 3.1 were made with melt flowing through single jets or, in Section 3.1.3 through single jets with an appropriately placed "upstream solid body".

\section{Experimental}

The experimental arrangement was described in a previous paper [4]. In brief, the block contain- ing the jets was attached to an Instron rheometer with the plunger of the rheometer pushing the melt through the capillaries. The orifices were 1.0 and $0.35 \mathrm{~mm}$ in diameter. For the single jet experiment (Section 3.1) one of the jets was removed and the exit plugged, for experiments (Section 3.1.3) an appropriate upstream obstruction was introduced by attaching it to a blank bolt. The rate of plunger movement and pressure could be controlled and recorded by the usual facilities of the Instron. Observations were made through glass windows and viewed by means of a polarizing microscope. High density polyethylene (Marlere 6002) was used for all experiments.

\section{Observations}

\subsection{Fibre formation in flowing melts}

3.1.1. Flow through unobstructed single jets

In the sequence of photographs shown in Fig. 1, the behaviour of molten polymer flowing into a single orifice is shown. The diameter of the orifice is $0.35 \mathrm{~mm}$ and the length of the hole downstream from the entrance is $1 \mathrm{~mm}$. Fig. 1 shows a series of photographs taken at a temperature of $140^{\circ} \mathrm{C}$ for a mean velocity $\bar{V}_{\mathrm{e}}$ into the orifice of $2.6 \mathrm{~cm}$ $\mathrm{sec}^{-1}$ (operating pressure within the cell $\sim 50$ bar). The photographs are taken between diagonally crossed polaroids, showing the development of crystallization as a function of time. Fig. 1a corresponds to immediately after flow has started where a symmetrical flow birefringence pattern is seen which relates to the local molecular orientation present in the melt near the entrance of the orifice. After a short 

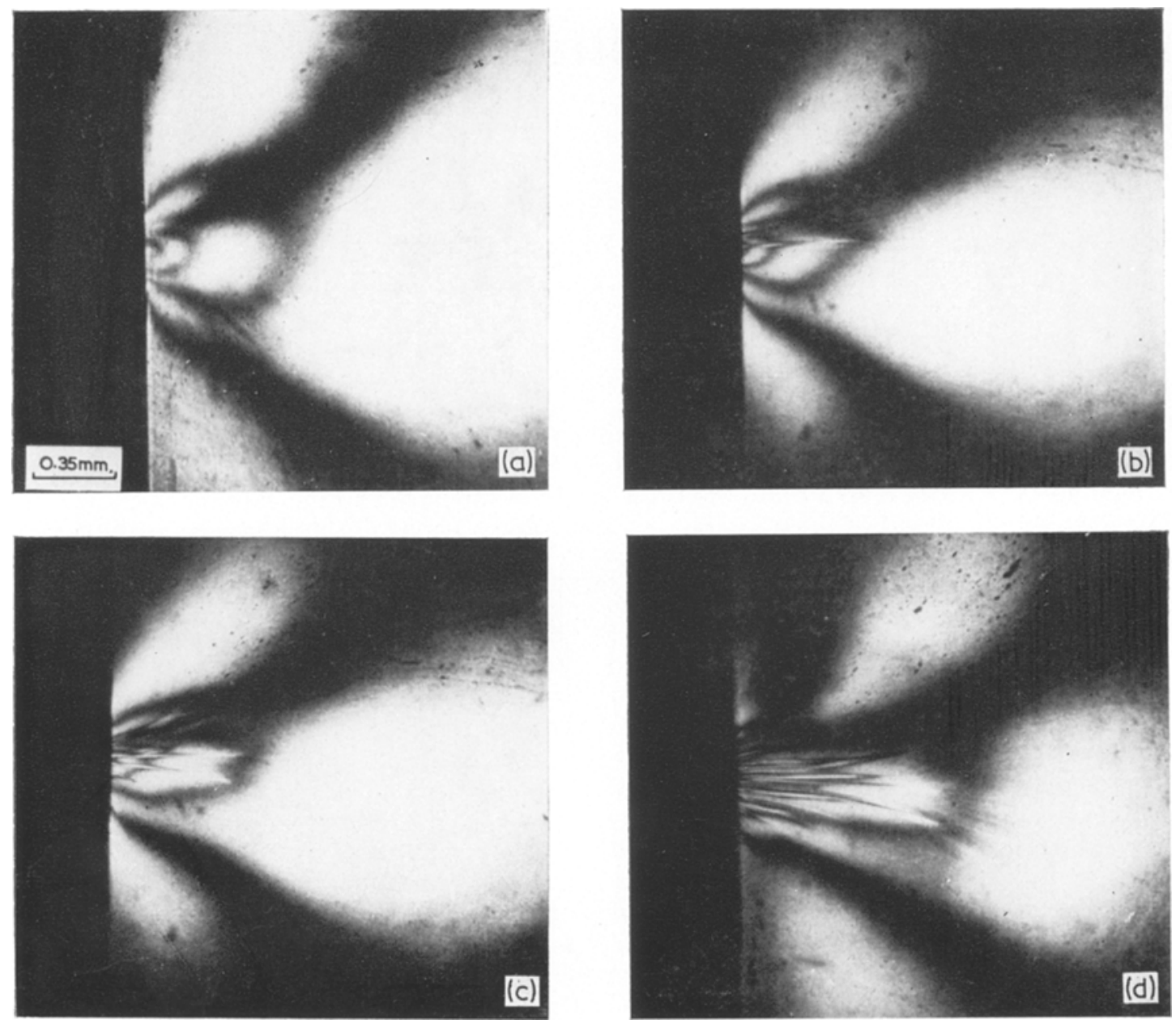

Figure 1 Photographs viewed between diagonally crossed polaroids showing the development with time of fibrous crystallization $T=140^{\circ} \mathrm{C}$. $V_{\mathrm{e}}=2.6 \mathrm{~cm} \mathrm{sec}^{-1}$.

time, usually of the order 1 to $5 \mathrm{sec}$, individual or groups of fibrous crystals appear in the region immediately upstream of the orifice (Fig. 1b). The tips of these crystals are then seen to migrate upstream to a position near the outer boundary of the birefringent region as shown in Fig. 1c and $d$. Migration of the tip then stops and a steady dynamic equilibrium state is achieved.

The diameter of the fibrous crystals observed varied between 5 and $50 \mu \mathrm{m}$; the number of observed fibres is also rather variable, and typically between 1 and 20 . On the cessation of flow, the fibres will disappear into the orifice as the flow rate decreases.

It should be stressed that this dynamic equilibrium situation is only present for a specific set of conditions. Both higher temperature and/or lower flow rate, will result in no crystallization occurring; lower temperature and/or higher flow rate will result in massive crystallization, where blocking and instabilities will set in.

Optical sections of the extrudate and X-ray observations of the material extruded through the orifice show that fibrous crystals are present in the extruded material, thus indicating that the fibres must be continuously produced in the region of the orifice. (This latter material will be presented in a later publication.)

The steady state situation, described above, can be best understood with the aid of Figs. 2, 3 and 4. In Fig. 2, the approximate external flow field is indicated for polymer flowing into a single orifice, showing that the fluid velocity increased rapidly as the entrance to the jet is approached. 


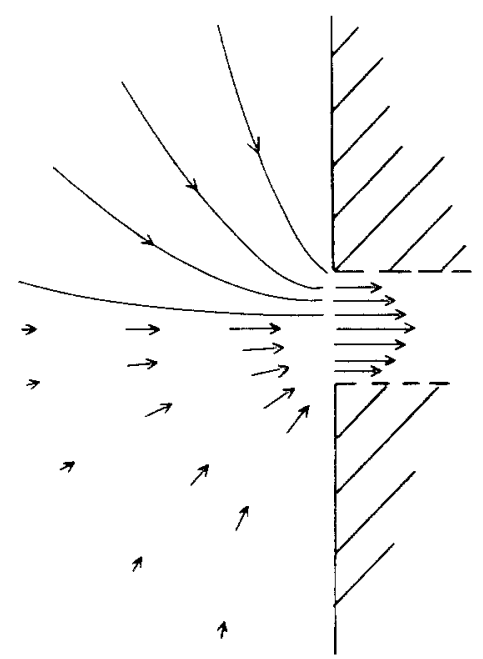

Figure 2 Schematic representation of flow into single orifice.

This is essentially extensional flow reaching a maximum strain of about $50 \mathrm{sec}^{-1}$.

Nucleation of the fibrous crystallization is observed to take place near the entrance of the orifice. The observation is consistent with the belief that flow induced fibrous crystallization occurs when polymer chains are aligned by the applied velocity gradient prior to crystallization; the degree of pre-alignment will be highest near the jet orifice.

Once the fibre has been nucleated it must grow continuously at the tip with the crystallized fibre being swept down by the flow into the orifice. In Fig. 1d we see that the tips of the fibres have migrated to regions of low melt velocity where the velocity difference between the moving fibre and effectively stationary polymer melt is large. The real situation at the growing tip corresponds to that shown in Fig. 3. A dynamic equilibrium situation is reached where the rate of generation of fibrous crystals equals the rate of loss of crystal into the orifice.

The flow field present at the tip can be more readily visualized by considering the fibre to be stationary. We then have streaming parallel to the fibre as shown schematically in Fig. 4. This is the "local" flow field that the growing fibre tip once nucleated will experience.

The above phenomena bear a striking resemblance to growth conditions pertaining to flow induced crystallization from solution. As known frominvestigations of Pennings (cited in [5])elongationalflow in solutions produces fibrous crystals; the shish-kebabs so-called because of their

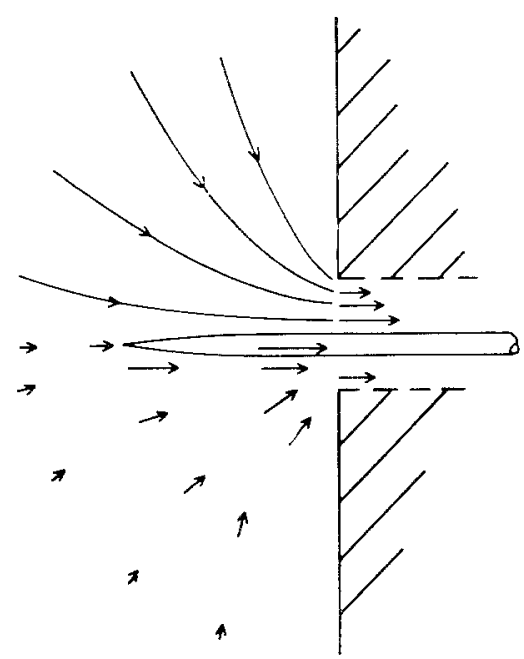

Figure 3 Schematic representation of flow field when a growing fibre is present.

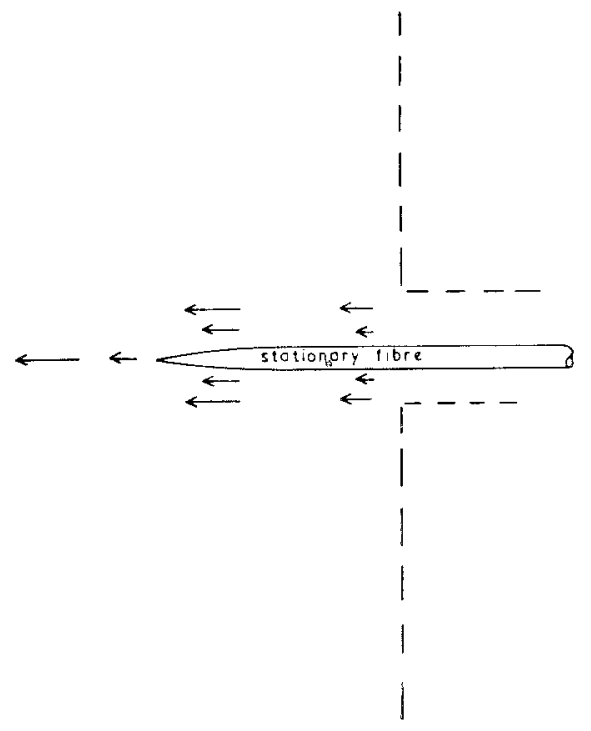

Figure 4 Schematic representation of local flow field present around growing fibre considering the fibre to be stationary.

composite fibre platelet microstructure. More recently, it has been reported that the elongational flow field is required for nucleation of these shish-kebabs but their continued growth can proceed under certain conditions even in weak simple shear flow [6]. Mackley [3] has accounted for this latter effect by considering the local flow field brought into being by streaming around the newly formed stationary fibre. Accordingly there will be a strong longitudinal velocity gradient at the tip which will elongate 
molecules locally and ensure the growth of the fibre. We see that the same type of flow field appears to control hydrodynamically induced fibrous crystal growth in the melt as shown by Fig. 4.

Much of the theoretical analysis adopted for the examination of the local flow field around a growing shish-kebab [3] can be carried over to study the local flow around a fibre in the melt. The analysis makes drastic assumptions in considering the fibre to be that of a rigid prolate ellipsoid of very high aspect ratio, it also considers the case of uniform streaming "Stokes flow" parallel to the major axis of the ellipsoid. Both assumptions seem acceptable for the melt as a first order approximation.

Using the equations derived previously [3] and substituting for parameters relating to the melt, we can derive the velocity field around the ellipsoid. We choose $U$, the stream velocity, so that $U=\bar{V}_{\mathrm{e}}=2.6 \mathrm{~cm} \mathrm{sec}-1$. The choice of the semi-minor axis $b$ of the ellipsoid is given as 10 $\mu \mathrm{m}$ corresponding to a typical observed halfwidth of the fibres. The choice of the length of the ellipsoid is somewhat less definite: however, provided this is very large in relation to $b$ it does not significantly affect the result. We choose the semi-major axis length $a=500 \mu \mathrm{m}$ giving an ellipsoid aspect ratio of 50 . Using these parameters the velocity along the symmetry axis downstream from the tip can be calculated as a function of distance from the tip and is given in Table I.

TABLE I Velocity along symmetry axis of fibre (in streaming direction)

\begin{tabular}{ll}
\hline $\begin{array}{l}\text { Distance from tip } \\
(\mathrm{cm})\end{array}$ & $\begin{array}{l}\text { Velocity as a fraction of stream } \\
\text { velocity, } U\end{array}$ \\
\hline 0 & 0 \\
$10^{-4}$ & 0.16 \\
$10^{-3}$ & 0.44 \\
$10^{-2}$ & 0.72 \\
$10^{-1}$ & 0.92 \\
\hline
\end{tabular}

From these results we see that in the region of the tip the velocity changes from zero at the tip to 0.44 of the stream velocity in a distance of 10 $\mu \mathrm{m}$. This means that a longitudinal velocity gradient of approximate magnitude $1.2 \times 10^{3}$ $\mathrm{sec}^{-1}$ must be operating at the tip for a stream velocity of $\bar{V}_{\mathrm{e}}=2.6 \mathrm{~cm} \mathrm{sec}^{-1}$. It is proposed that this longitudinal velocity gradient at the tip is of sufficient magnitude to stretch polymer chains and thereby promote the growth of fibrous crystals at the tip of the fibre.

\subsubsection{On the existence of a stable dynamic equilibrium state}

When there is a crystal fibre in the exit pipe, which grows upstream from the orifice, there are two distinct characteristics of the flow field which tend to produce molecular alignment and therefore to promote crystallization. One is the longitudinal velocity gradient related to the general convergent flow towards the orifice; here the streamlines of the flow (with the exception of regions very close to the orifice) are essentially directed along the radius towards the centre point of the orifice, with a stream velocity, $V_{\mathrm{r}}$, which averages over the hemisphere to $\bar{V}_{\mathrm{r}}=$ $\bar{V}_{\mathrm{e}}\left(a^{2} / 2 r^{2}\right)$ where $\bar{V}_{\mathrm{e}}$ is the mean velocity in the exit pipe, the radius of which is $a$. $V_{\mathrm{r}}$ is zero on the walls at $90^{\circ}$ from the symmetry axis and maximum on the axis; over a fairly wide solid angle around the axis it will be approximately uniform and approximately equal to $\bar{V}_{\mathrm{e}}\left(a^{2} / r^{2}\right)$ for, say, $r>a$. In, and close to the exit pipe, say, for $r<a$ the velocity tends to a constant. An adequate approximate representation of this behaviour is given by a

$$
V_{\mathrm{r}}=\frac{\bar{V}_{\mathrm{e}} 5 a^{2}}{\left(r^{2}+5 a^{2}\right)} .
$$

The longitudinal strain-rate is then given by

$$
\dot{\epsilon}_{\text {gen }}=-\frac{\partial V_{\mathrm{r}}}{\partial r}=\bar{V}_{\mathrm{e}}\left(\frac{10 a_{\mathrm{r}}^{2}}{\left(r^{2}+5 a^{2}\right)^{2}}\right)
$$

with $\bar{V}_{\mathrm{e}}=2.6 \mathrm{~cm} \mathrm{sec}^{-1}$ and $a=0.18 \mathrm{~mm} \dot{\epsilon}_{\text {gen }}$ is a maximum at $r=5 a / 3$ having a value $\dot{\epsilon}_{\text {gen }}=48$ $\mathrm{sec}^{-1}$ falling off as $r^{-3}$ for larger values of $r$.

The second characteristic of the flow field tending to produce molecular alignment is the elongational flow at the tip of the fibre as discussed in the previous section, attributable to the relative motion of the fibre tip and ambient fluid. It may be assumed that the fibre moves longitudinally with a velocity $\alpha \bar{V}_{\mathrm{e}}$ appropriate to its position in the exit pipe where $0<\alpha<2$. Thus the hydrodynamic relative velocity of the fibre is

$V_{\mathrm{reI}}=\alpha \bar{V}_{\mathrm{e}}-V_{\mathbf{r}} \sim \bar{V}_{\mathrm{e}}\left(\alpha-\frac{5 a^{2}}{r^{2}+5 a^{2}}\right)$.

This relative motion produces an elongational flow on the axis of the fibre, close to its tip, with a local strain rate of order of magnitude 


$$
\dot{\epsilon}_{\mathrm{loc}}=\frac{V_{\mathrm{rel}}}{x}=\frac{\bar{V}_{\mathrm{e}}}{x}\left(\alpha-\frac{5 a^{2}}{\left(r^{2}+5 a^{2}\right)}\right)
$$

where $x$ is a characteristic distance. If we choose $x$ as being equal to the diameter of the fibre this will give a strain rate value equal to that calculated in the previous section i.e. when $r \gg a$ and $V_{\text {rel }}$ approaches its maximum value $V_{\text {rel }}=$ $2.6 \mathrm{~cm} \mathrm{sec}^{-1}$, also $x=20 \mu \mathrm{m}$ then $\dot{\epsilon}_{\mathrm{loc}}=1300$ $\mathrm{sec}^{-1}$.

We note that the maximum local strain-rate at the tip is an order of magnitude larger than the maximum strain-rate of the general convergent flow and one might, therefore, well suppose that once nucleation has occurred and the fibre formed the general convergent flow has little further significance for subsequent growth. The observations made here show, however, that this is not the case. If the strain-rate $\dot{\epsilon}_{10 c}$ attributable to $V_{\text {rel }}$ were the only significant factor, one would expect the rate of fibre elongation $V_{\text {cryst }}$ to be a monotone increasing function of $\dot{\epsilon}_{10 c}$ which itself according to Equation 4 is a monotone increasing function of $r$. The fibre is either sucked away downstream and is lost, or grows further upstream, according as $\left(V_{\text {cryst }}-\alpha \bar{V}_{\mathrm{e}}\right)$ is less or greater than zero. With $V_{\text {cryst }}$ dependent only on $\dot{\epsilon}_{\text {loe }}$ there can be no stable dynamic equilibrium: there can be one value of $r, r_{\mathfrak{c}}$ such that fibres not extending as far as $r_{\mathrm{c}}$ are sucked away and lost, and those extending beyond grow without limit.

The observations of Fig. 1d show that there is a range of flow conditions in which there is dynamic equilibrium, the fibre growth balancing the fibre retraction rate so as to maintain a constant length of fibre $r$ upstream from the orifice. This is only possible if for some range of $r$

$$
V_{\text {cryst }}-\alpha V_{e}=0
$$

also

$$
\frac{\partial V_{\text {cryst }}}{\partial r}<0
$$

where a perturbation increasing the length of fibre will decrease $V_{\text {cryst }}$ and a perturbation decreasing the length of fibre will increase $V_{\text {cryst. }}$

This dynamic equilibrium situation can exist if both aligning features of the flow field contribute in promoting crystallization, e.g. additivity though not necessarily with equal coefficients as

$$
\begin{gathered}
V_{\text {cryst }}=A \dot{\epsilon}_{1 \mathrm{oc}}+B \dot{\epsilon}_{\mathrm{gen}} \\
=A \frac{\bar{V}_{\mathrm{e}}}{x}\left(\alpha-\frac{5 a^{2}}{\left(r^{2}+5 a^{2}\right)}\right) \\
+B \bar{V}_{\mathrm{e}} \cdot \frac{10 a^{2} r}{\left(r^{2}+5 a^{2}\right)^{2}} .
\end{gathered}
$$

Both individual contributions and final form of Equation 5 are shown in Fig. 5.

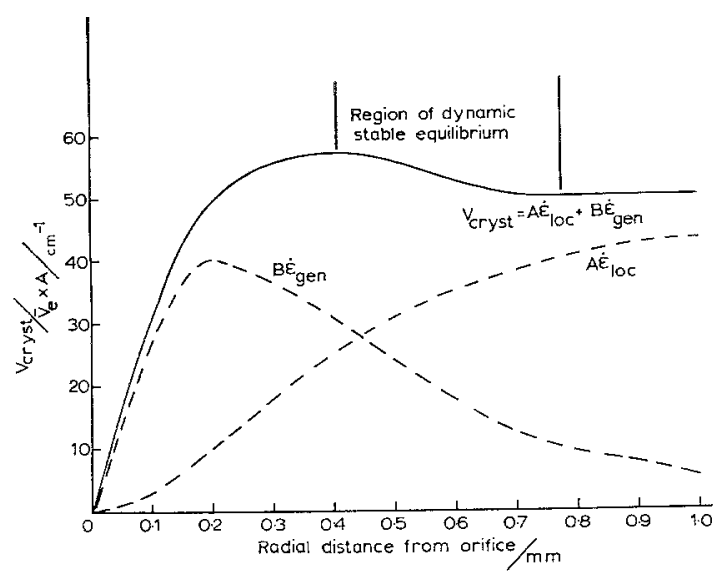

Figure 5 Graph of $V_{\text {cryst }} / \bar{V}_{\mathrm{e}} A$ against $r$ where $\alpha=1, a=$ $0.18 \mathrm{~mm}, x=2 \times 10^{-2} \mathrm{~mm}$ and $B=25 A$.

Experimentally, we observe a dynamic equilibrium at $r$ equal to about $4 a$. To achieve this, suitable values of $A$ and $B$ must be chosen. The differential of Equation 5 is

$$
\begin{aligned}
\frac{\partial V_{\text {eryst }}}{\partial r}= & \frac{10 \bar{V}_{\mathrm{e}} a^{2}}{\left(r^{2}+5 a^{2}\right)^{2}} \\
& \left\{\frac{A r}{x}+B\left(1-\frac{4 r^{2}}{\left(r^{2}+5 a^{2}\right)^{2}}\right)\right\} .
\end{aligned}
$$

From this we obtain a maximum value of $V_{\text {cryst }}$ at $r=4 a$ if $B=17.6 \mathrm{~A}$. The maximum is followed by a region for $r>4 a$ where ( $r V_{\text {cryst }} /$ $\partial r)<0$, which is a necessary condition for a region of stable dynamic equilibrium. Values of $B$ less than $17.6 A$ will either not give a region of stable equilibrium or alternatively the equilibrium range will not correspond to the experimentally observed range. Values of $B$ greater than $17.6 B$ will increase the range of dynamic stable equilibrium. For clarity we choose $B=25 A$ in Fig. 5 which shows clearly the range where a dynamic stable equilibrium exists. By a suitable choice of $A$ we can arrange that the condition $V_{\text {cryst }}-\bar{V}_{\mathrm{e}}=0$ ( $\alpha$ taken as being equal to 1) is satisfied for the region indicated in Fig. 5 where $\left(\partial V_{\text {cryst }} / \partial r\right)<0$. From this examina- 
tion we are forced to accept that the effect of $\dot{\epsilon}_{\text {gen }}$ on crystal growth must be enhanced relative to that of $\dot{\epsilon}_{\text {loc }}$ by a factor of at least 17.6 for a stable equilibrium situation to exist in the region $r=4 a$.

It may be possible to account for the enhanced effect of $\dot{\epsilon}_{\text {gen }}$ by noting that time effects may be important; the relative weakness of $\dot{\epsilon}_{\text {gen }}$ may be countered by the increased time molecules spend in this velocity gradient compared to the local velocity gradient at the tip. Also if attachment followed by orientation is involved in the formation of the fibrous crystals, as proposed for the case of shish-kebabs [3] it can be visualized that weak pre-orientation of molecules can significantly affect attachment rates and hence overall growth rates. In this case we imagine the general velocity gradient weakly pre-aligning the molecules, which enhances the attachment rate onto the crystal face. Once molecules become attached they have the ability to become aligned at the tip by the large local longitudinal velocity gradient operating at the tip, fibrous crystal growth thus proceeding from this region.

As implied by the above mechanistic discussion the way in which the two aligning phenomena in the flow field combine may well be more complex than the simple additive combination of Equation 5; this is the simplest of suppositions for a way of combining the two distinct aligning effects which is adequate to account for the observed phenomenon, with a range of conditions giving a stable dynamic equilibrium in the growth of the fibres.

To conclude, we observe that convergent flow into a single orifice is capable under certain conditions of nucleating fibrous crystals near the orifice. Once the fibre is formed theoretical analysis suggests that the local velocity gradient at the tip of the fibre will control further growth; however, the observation of a stable dynamic equilibrium position implies that the weak orientation of the convergent flow at the corresponding large distance from the orifice has a continuing influence on crystal growth.

\subsubsection{Flow into a single orifice with an upstream obstruction}

In the sequence of photographs shown in Fig. 6 the behaviour of molten polyethylene flowing into a single orifice with an upstream needle-like obstruction is shown. The diameter of the orifice and the length of the hole downstream from the entrance are both $1 \mathrm{~mm}$. The upstream
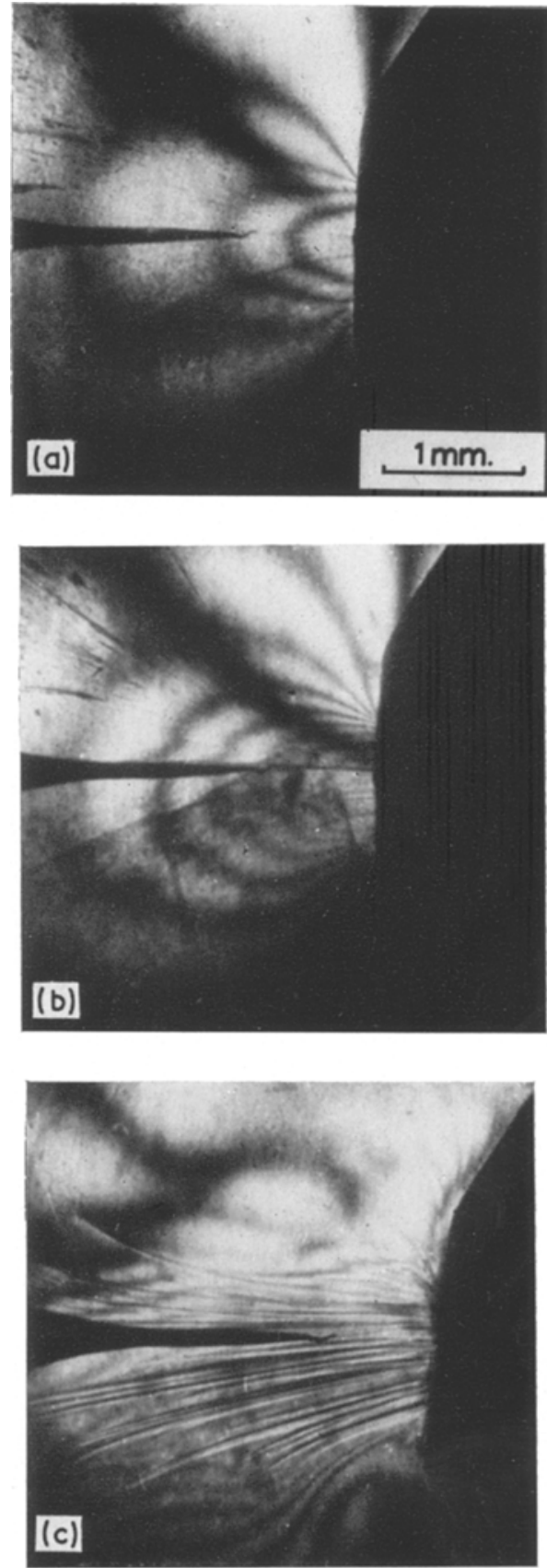

Figure 6 Photographs viewed between diagonally crossed polaroids showing the development of fibrous crystallization for molten polethylene flowing into an orifice with a stationary needle-like obstruction upstream, $T=140^{\circ} \mathrm{C}$. (a) $V_{\mathrm{e}}=0.8 \mathrm{~cm} \mathrm{sec}^{-1}$, (b) $V_{\mathrm{e}}=3.3 \mathrm{~cm} \mathrm{sec}^{-1}$, (c) $V_{\mathrm{e}}=$ $8.0 \mathrm{~cm} \mathrm{sec}^{-1}$.

needle-like obstruction was made from steel. Fig. 6 shows a series of photographs taken at a 
temperature of $140^{\circ} \mathrm{C}$. The photographs are taken between diagonally crossed polaroids showing the development of crystallization as a function of increasing flow velocity.

Fig. 6a shows the case for a mean velocity into the orifice of $\bar{V}_{\mathrm{e}}=0.8 \mathrm{~cm} \mathrm{sec}-1$. In this picture the familiar flow birefringence picture is seen relating to the oriented melt entering the orifice. A slight discontinuity is visibie immediately downstream from the tip but the general behaviour is essentially the same as if the upstream obstruction was not present. Fig. 6 b shows the situation for enhanced velocity $\bar{V}_{\mathrm{e}}=3.3 \mathrm{~cm}$ $\mathrm{sec}^{-1}$, where it can now clearly be seen that fibrous crystallization has occurred appearing to originate from the tip of the 'needle'. We conclude that the local flow around the tip of the obstruction has the ability to nucleate the fibrous crystal, whereas the normal flow into the jet has not. Fig. $6 \mathrm{c}$ shows the case for a further increase in the melt velocity $\bar{V}_{\mathrm{e}}=8.0 \mathrm{~cm} \mathrm{sec}-1$. This picture resembles that of Fig. 1c and $d$ in the single orifice analysis; here the external flow field has nucleated fibrous crystals over the whole observed volume upstream of the orifice and growth continues in the same way as discussed in Section 3.1.2.

From this present series of photographs we can conclude that melt streaming around a needlelike obstruction can preferentially nucleate fibrous crystallization; thus we have developed a way of controlling nucleation. Also the fact that this type of flow field causes nucleation supports the proposal for the mechanism explaining the continued growth of the fibres discussed in Section 3.1.2. In this present case the flow around the metal needle can cause nucleation at the tip, presumably due to the presence of a high local longitudinal velocity gradient stretching the chains in the region of the tip. In a similar way it was proposed in Section 3.1.2 that the fibrous crystal growth can be maintained by the streaming of molten polymer around the crystal tip thereby producing localized chain stretching at the tip.

\subsection{Blockage of melt flow in a double jet under enhanced pressure}

It has been reported (e.g. [7-9]) that melts when forced through a capillary, as done in the course of conventional rheological tests, can solidify at temperatures above their normal melting points and that the resulting solidified products, when extracted from the capillaries, can display remarkable properties such as unusually high melting point, Young's modulus and optical transparency. It is generally believed that chain orientation due to flow is involved. With this background in mind we carried out experiments designed to create blockage and to observe the process visually while it is occurring.

The mutually opposed double jet apparatus of earlier works [4] was used for this purpose as here the effects to be described occurred with greatest clarity and readiness. The jet diameter was $1 \mathrm{~mm}$ but the jet length was increased by a factor of 30 , to $3 \mathrm{~cm}$. This enabled the pressure to be raised to 500 bars from the 50 bar applied in previous experiments.

Fig. 7 shows a series of photographs taken as a function of time for polyethylene flowing into the opposed orifices at a temperature of $140^{\circ} \mathrm{C}$. The photographs are taken in bright-field illumination rather than crossed polaroids to assist in the clarity of interpretation. It is not possible to assign a single value to the flow velocity into the orifices as this changes with time.

Fig. 7a corresponds to the zero time case immediately after the plunger, forcing the melt into the orifices, has started advancing at a constant rate. As soon as the flow into the orifices has become established with an approximate orifice entry velocity of $3.0 \mathrm{~cm} \mathrm{sec}-1$ and corresponding pressure of about 100 bar a discrete fibrous crystal is observed to form on the symmetry axis of the system, Fig. $7 \mathrm{~b}$ and c. At this point the pressure rises rapidly and flow effectively stops. After a short time, typically about $10 \mathrm{sec}$, a second finer scale fibrous crystallization is seen to originate from one or both of the jets as shown in Fig. 7d; the melt pressure is now about 500 bar. The fine scale fibrous crystals rapidly grow completely obscuring the transmitted light within $30 \mathrm{sec}$ of first appearing at the orifices (Fig. 7f). At this stage complete blockage has occurred and the plunger advance must be stopped to prevent the explosion of the glass observation windows.

If the pressure is then removed by reversing the direction of advance of the plunger, the fine scale pressure-induced fibrous crystallization is seen to melt (Fig. $7 \mathrm{~g}$ and $\mathrm{h}$ ) leaving the original flow-induced fibrous crystal. The buckling of the flow induced crystal is due to a slight reverse flow present when the plunger direction is reversed.

In this situation, crystallization has taken place in two distinct forms. Initially, when flow was present a flow-induced fibrous crystal was 

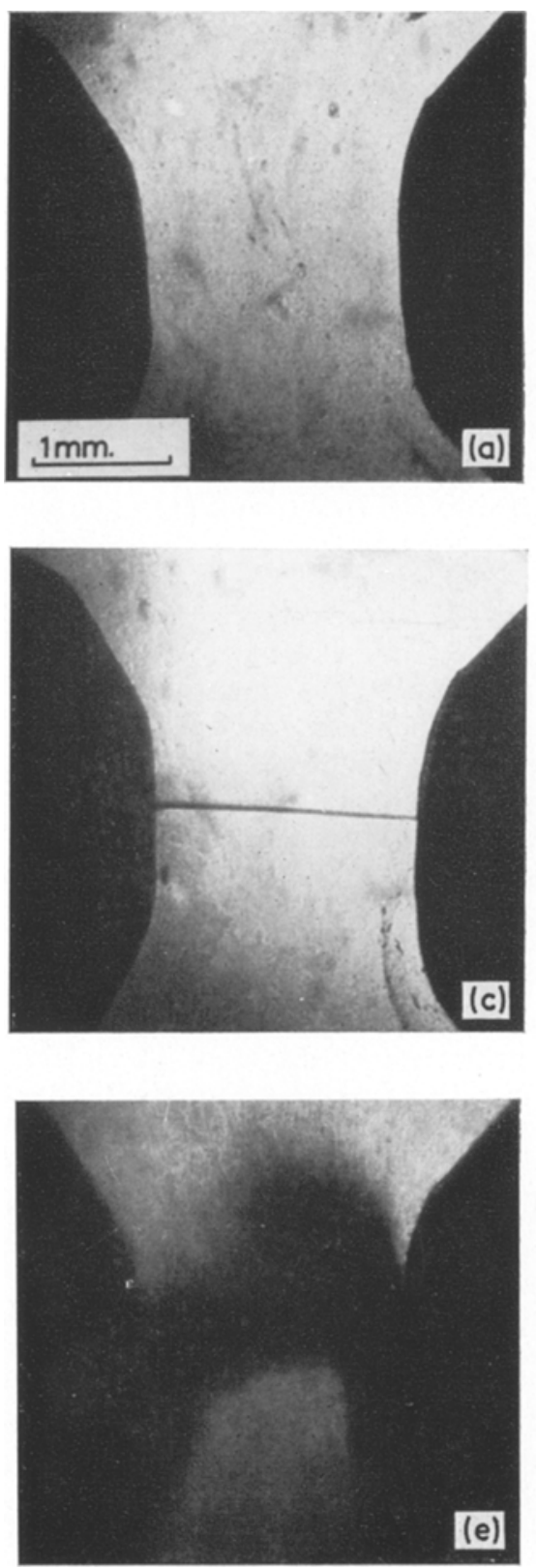

Figure 7

formed. Later, when flow had effectively stopped and the pressure risen, a pressure-induced fibrous crystal was formed. On removal of pressure the pressure-induced component melted leaving the flow-induced component. The striking difference in melting behaviour of the two crystal types suggest that the structures of these crystals are fundamentally different to one another; the
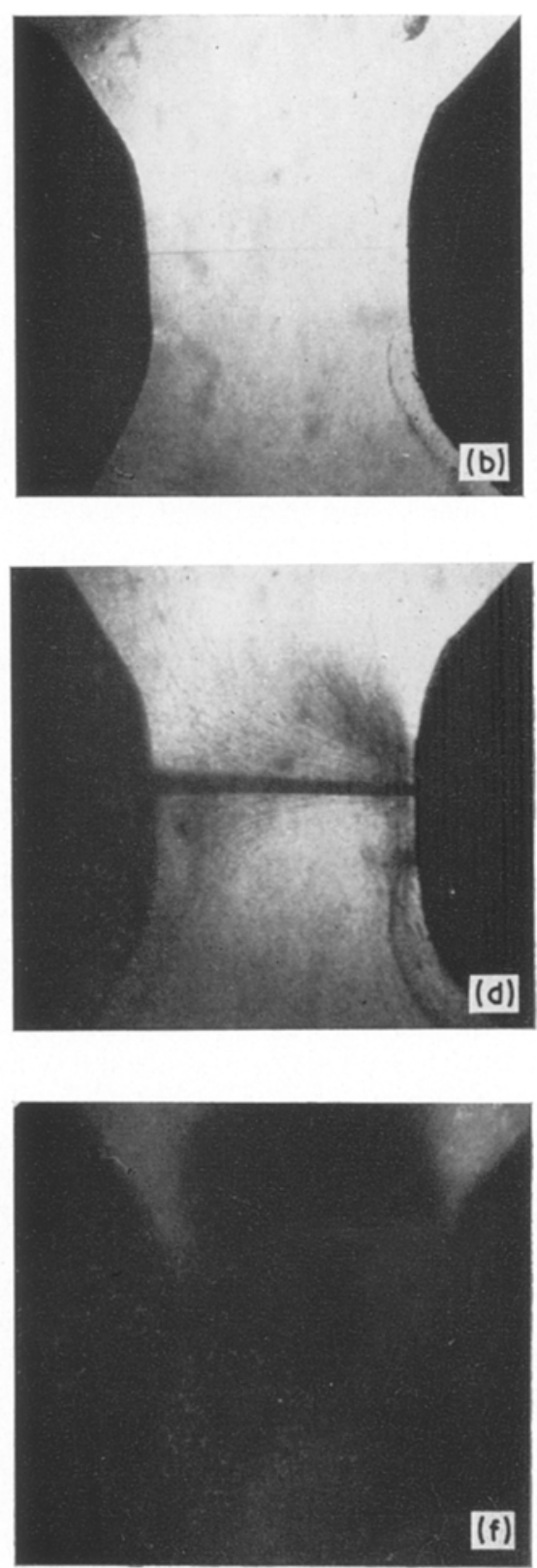

greater stability of the flow-induced fibrous crystal implies that this crystal type is the more perfect of the two.

\section{Conclusions}

Our observations illustrate the importance of longitudinal velocity gradients in both nucleation and growth of fibrous crystals from the melt. We 

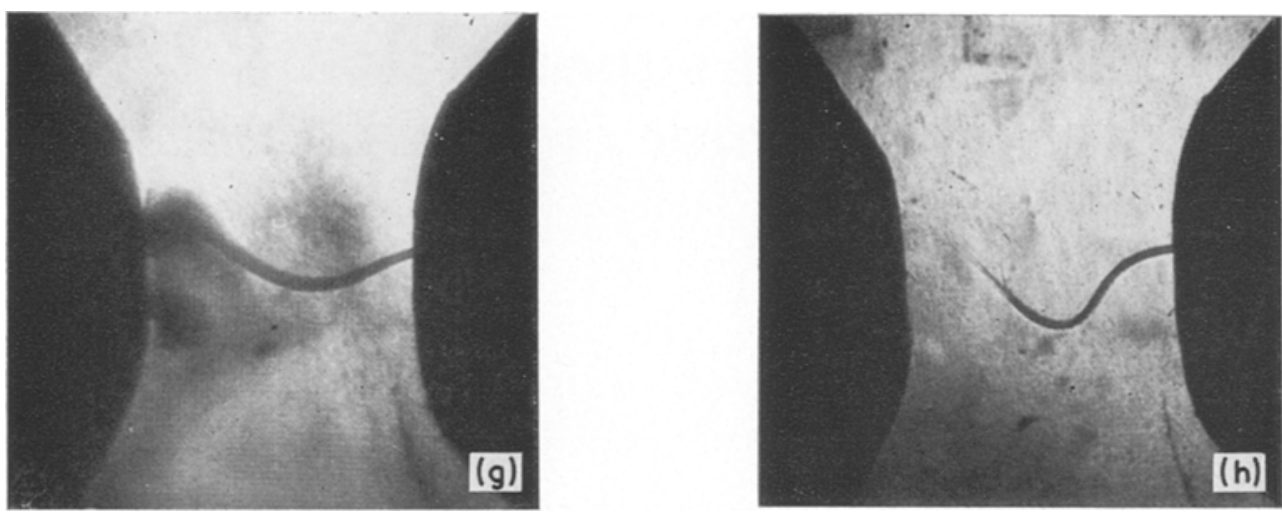

Figure 7 Bright-field photographs showing the development of flow- and pressure-induced crystallization between opposed orifices $T=140^{\circ} \mathrm{C}$.

have shown that for a narrow range of conditions it is possible to generate fibrous crystals continuously in a dynamic equilibrium state by flowing polyethylene melt into an orifice. Nucleation of the fibres is controlled by the longitudinal velocity gradient $\dot{\epsilon}_{\text {gen }}$ near the orifice due to the accelerating flow into the orifice and subsequent growth is then controlled by a combination of $\dot{\epsilon}_{\text {gen }}$ and the local longitudinal velocity gradient at the tip of the fibre $\dot{\epsilon}_{10 c}$. Further it has been shown that nucleation of fibrous crystals can be induced and controlled by the introduction of a needle-like obstruction in the flow, which underlines the principle of local chain extension at the downstream tip of a body and also illustrates the effects obstacles deliberately introduced or unintentionally present can have on the processing of polymer melts particularly when not much above the melting temperature. Finally, our observations of jet blocking due to crystallization show that two distinct types of fibrous crystals are involved. A flow-induced fibrous crystal which is stable at all pressures and a pressure-induced fibrous crystal which at the temperature of formation is only stable at elevated pressures.

Observations of the kind reported here should be of relevance to current extrusion and spinning procedures as well as to possible new polymer processing techniques.

\section{Acknowledgement}

We would like to thank Dr C. G. Cannon, I.C.I. Pontypool for his valuable help and advice in the early stages of this work. We would also like to thank Dr D. Grubb for his assistance in examining some optical sections of the extrudates. One of us (M.R.M.) wishes to acknowledge the generous financial support of the I.C.I. fellowship fund.

\section{References}

1. M. R. MACKLEY and A. Keller, Phil. Trans. Roy. Soc. (Lond.) 278 (1276) (1975) 29.

2. M. R. MACKLEY, Colloid and Polymer Sci, 253 (1975) 261.

3. Idem, ibid, 253 (1975) 373.

4. M. R. MACKLEY and A. Keller, Polymer 14 (1973) 16.

5. A. Keller and M. R. MACKLEy, Pure Appl. Chem. 34 (1974) 1

6. A.J.PENNINGS, A. ZWYNEBURG andR. LAGEVEEN, Kolloid Z. u. Z. Polymere 251 (1973) 500.

7. A. K. VAN DER VEGT and P. P. A. SMIT, $A d v$. Polymer Sci. monograph 26 (1967) 313.

8. J. H. southern and R. S. Porter, J. Appl. Polymer Sci. 14 (1970) 2305.

9. T. NIIKUNI and R. S. PORTER, J. Mater. Sci. 9 (1974) 389.

Received 31 January and accepted 17 February 1975. 